OPEN ACCESS

Edited by: Alberto Rainer,

Campus Bio-Medico University, Italy

Reviewed by:

Chris Boesch,

University of Bern, Switzerland Mark Christopher Turner, Coventry University, United Kingdom

*Correspondence: Luigi Ferrucci

FerrucciLu@grc.nia.nih.gov

Richard G. Spencer

spencerri@mail.nih.gov

${ }^{t}$ These authors have contributed equally to this work

Specialty section:

This article was submitted to Integrative Physiology, a section of the journal Frontiers in Physiology

Received: 23 November 2020 Accepted: 21 April 2021 Published: 30 June 2021

Citation:

Brennan NA, Fishbein KW, Reiter DA, Ferrucci $L$ and Spencer RG (2021) Contribution of Intramyocellular Lipids to Decreased Computed

Tomography Muscle Density With Age. Front. Physiol. 12:632642. doi: 10.3389/fphys.2021.632642

\section{Contribution of Intramyocellular Lipids to Decreased Computed Tomography Muscle Density With Age}

\author{
Nicholas A. Brennan1, Kenneth W. Fishbein', David A. Reiter ${ }^{2}$, Luigi Ferrucci ${ }^{* *}$ and \\ Richard G. Spencer ${ }^{* \dagger}$ \\ 1 Laboratory of Clinical Investigation, NIA, NIH, Baltimore, MD, United States, ${ }^{2}$ Department of Radiology and Imaging \\ Sciences, Emory University School of Medicine, Atlanta, GA, United States, ${ }^{3}$ Longitudinal Studies Section, NIA, NIH, \\ Baltimore, MD, United States
}

Skeletal muscle density, as determined by computed tomography (CT), has been shown to decline with age, resulting in increased frailty and morbidity. However, the mechanism underlying this decrease in muscle density remains elusive. We sought to investigate the role of intramyocellular lipid (IMCL) accumulation in the age-related decline in muscle density. Muscle density was measured using computerized tomography (CT), and IMCL content was quantified using in vivo proton magnetic resonance spectroscopy $\left({ }^{1} \mathrm{H}-\mathrm{MRS}\right)$. The study population consisted of 314 healthy participants (142 men, 32-98 years) of the Baltimore Longitudinal Study of Aging (BLSA). In addition to IMCL quantification, obesity-related covariates were measured, including body mass index (BMI), waist circumference, and circulating triglyceride concentration. Higher IMCL concentrations were significantly correlated with lower muscle density in older individuals, independent of age, sex, race, and the obesity-associated covariates $(p<0.01)$. Lower muscle density was also significantly associated with greater ageadjusted IMCL, a variable we constructed using LOESS regression $(p<0.05)$. Our results suggest that the accumulation of IMCL may be associated with a decrease in muscle density. This may serve to define a potential therapeutic target for treatment of age-associated decreased muscle function.

\footnotetext{
Keywords: muscle, aging, proton magnetic resonance spectroscopy, intramyocellular lipids, computerized tomography
}

\section{INTRODUCTION}

Skeletal muscle radiological density, as assessed with computerized tomography (CT), has been shown to decrease with age (Lauretani et al., 2006). Independent of age and other potential confounders, lower muscle density has been associated with frailty (Cesari et al., 2006), mobility limitations (Visser et al., 2005; McDermott et al., 2009), increased risk of hip fracture (Lang et al., 2010), poor lower extremity performance (Visser et al., 2002; Hicks et al., 2005; Cawthon et al., 2009), and lower muscle quality, defined as the specific force generated per unit of muscle volume (Goodpaster et al., 2001a; Conroy et al., 2012). In addition, lower muscle density has been associated with metabolic outcomes such as loss of oxidative enzyme capacity (SchrauwenHinderling et al., 2007), insulin resistance, the metabolic syndrome and higher intermuscular fat deposition (Goodpaster et al., 2000a, 2001a; Visser et al., 2005; Addison et al., 2014). Based on these 
data, it has been suggested that low muscle density is a biomarker of impaired metabolism and poor health status.

In spite of the consistent observation that muscle density declines with age, and the strong relationship between poor muscle density and adverse health outcomes (Pahor and Kritchevsky, 1998), the underlying mechanisms that cause reduction in muscle density remain poorly understood. Central hypotheses point to accumulation of small adipose cell aggregates that cannot be resolved by CT, but which affect overall tissue density. At the microscopic level, accumulation of lipids occurs mainly in two distinct compartments. First, intramyocellular lipid (IMCL) represents fat stored in the form of cytoplasmic droplets within myocytes; this can occur in response to metabolic derangements, including mitochondrial dysfunction. IMCL tends to be uniformly distributed throughout the muscle, and is particularly high in diabetic and obese individuals (SchrauwenHinderling et al., 2006; Weis et al., 2007; Aguer et al., 2010; Brumbaugh et al., 2012; Bredella et al., 2013; Noble et al., 2014). In contrast, extramyocellular lipids (EMCL) are layers of fat deposited outside myocytes. Unlike IMCL, EMCL is largely metabolically inert, and its quantification is highly dependent on voxel orientation and placement (Boesch et al., 1997). Therefore, our study focuses on IMCL deposition in aging muscle. While neither IMCL nor EMCL can be directly visualized with CT, macroscopic fat can be readily delineated. Additionally, large amounts of macroscopic fat can lead to a high EMCL signal, obscuring the ability to accurately discriminate between these two depots of fat using magnetic resonance spectroscopy.

Infiltration of muscle with fat is well-known to indicate decreased muscle quality. This can be observed with CT, with fat-infiltrated muscle demonstrating decreased attenuation as compared to non-fatty muscle (Prasetyo et al., 2020). However, this attenuation is not specific to the most metabolically significant fat component, IMCL. In contrast, MR spectroscopy provides a means to directly assess IMCL. Unfortunately, MRS is a highly specialized technique which is not widely available outside of medical research centers. Thus, we evaluate the degree to which IMCL contributes to the decreased CT attenuation exhibited by fat-infiltrated muscle. This provides insight into the degree to which CT evaluation of muscle can indicate decreased muscle quality as defined by IMCL infiltration.

The metabolic role of IMCL is recognized as providing a readily accessible energy reserve during high-demand exercise and, consistently, IMCL has been shown to increase with high-intensity training (Goodpaster et al., 2001b). Conversely, accumulation of intramyocellular fat droplets in other settings is attributed to the relative inability of dysfunctional mitochondria to fully process lipid substrates through beta oxidation. In accordance with this view, increased deposition of IMCL has been associated with oxidative stress and glucose intolerance. In addition, increased IMCL has been shown to correlate with insulin resistance in the obese (Pan et al., 1997; Goodpaster et al., 2000b) and older populations (Petersen et al., 2003; Cree et al., 2004; St-Onge, 2005; Nakagawa et al., 2007). Individuals with metabolic syndrome, characterized in part by insulin resistance, tend to have high IMCL (Perseghin, 2005; Ingram et al., 2011; Yokota et al., 2013). Finally, compared to normal weight individuals, obese patients with high IMCL exhibit decreased muscle force, diminished myofibril contraction rate, and decreased power production (Choi et al., 2016).

Studies of IMCL and EMCL have been greatly enhanced by application of magnetic resonance spectroscopy (MRS) and imaging (MRI) (van der Meer et al., 2012; Machann et al., 2013). Proton MRS ( $\left.{ }^{1} \mathrm{H}-\mathrm{MRS}\right)$ is currently the leading technique for quantifying IMCL in skeletal muscle. These fat compartments can be distinguished from each other based on the small difference in resonance frequency they exhibit within the magnetic field of an MR system (Schick et al., 1993; Boesch, 2007), leading to their visualization as resolved, or partly-resolved, resonances in high-quality ${ }^{1} \mathrm{H}$-MRS spectra.

Specifically defining muscle density as the mean CT attenuation coefficient of mid-thigh muscle tissue, we hypothesized that lower muscle density would be associated with accumulation of IMCL, rendering density a potential indicator of metabolic deficits. $1 \mathrm{H}$-MRS has been used extensively to quantify IMCL and EMCL in human subjects (Schick et al., 1993; Boesch et al., 1997; Jacob et al., 1999; Krssak et al., 1999; Hwang et al., 2001; Howald et al., 2002; Larson-Meyer et al., 2002), while CT muscle density has been incorporated into many studies of aging (Goodpaster et al., 2001a; Anderson et al., 2013; Aubrey et al., 2014; Kalyani et al., 2014). For example, Goodpaster et al. (2001a) found that higher muscle density is associated with higher specific force production and lower BMI, while (Aubrey et al., 2014) found that lower muscle attenuation is correlated with cancer progression and poor health outcomes.

Accordingly, in this study we sought to investigate the association between muscle density, as determined by CT (Goodpaster et al., 2001a; Visser et al., 2005), and IMCL, as determined by ${ }^{1} \mathrm{H}$-MRS using data collected from subjects enrolled in the Baltimore Longitudinal Study on Aging (Ferrucci, 2008), a well-characterized cohort of normatively aging adults. Specifically, we tested the hypothesis that the decrease in muscle density associated with aging is accounted for, at least in part, by the accumulation of IMCL. In addition, due to the documented association between IMCL and obesity, we investigated the individual correlations between IMCL and several markers of obesity, including BMI itself (Amin et al., 2015), serum cholesterol concentration (Miettinen, 1971; Kurata et al., 1990), waist circumference (Brumbaugh et al., 2012; Raja et al., 2014), which is also positively associated with cardiovascular disease (Akil and Ahmad, 2011) and type II diabetes (Golay and Ybarra, 2005), and triglycerides, a major correlate of adipose tissue and dietary fat intake in humans (Cox and Garcia-Palmieri, 1990).

\section{MATERIALS AND METHODS}

\section{Participants}

The study population, experimental design, and measurement protocols of the Baltimore Longitudinal Study of Aging (BLSA) have been previously reported (Ferrucci, 2008). The BLSA is a longitudinal cohort study established in 1958 and funded and conducted by the National Institute on Aging Intramural Research Program. The BLSA enrolls community-dwelling adults 
with no major chronic conditions or functional impairments. Usable ${ }^{1} \mathrm{H}$-MRS-based IMCL measurements (see below) were collected from 490 BLSA participants from November 2009 to September 2016. Of these, 314 participants had complete datasets including BMI, cholesterol, waist circumference, circulating triglycerides, and CT muscle attenuation measurements. Certified technicians administered all assessments using standardized protocols (Zane et al., 2017). The Institutional Review Board of the National Institute of Environmental Health Sciences approved the experimental protocol, and all participants provided written informed consent.

\section{Magnetic Resonance Spectroscopy}

Participants were placed feet first in a 3T Philips Achieva MR scanner (Philips, Best, The Netherlands) in a supine position. IMCL values were obtained from in vivo spectra of ${ }^{1} \mathrm{H}$-containing metabolites using the internal body coil for excitation. A pair of Flex-M coils was used for signal acquisition, with one element placed posteriorly and one anteriorly over the vastus medialis muscle of the left mid-thigh. Localized spectra were obtained using the PRESS sequence, with a voxel size of $8 \times 8 \times 40 \mathrm{~mm}$, with the third dimension being head-to-foot, an echo time of $55 \mathrm{~ms}$, a repetition time of $2,000 \mathrm{~ms}, 32$ signal averages, 4 step phase cycle, and a receiver bandwidth of 2,000 Hz. The number of complex data points in one scan, without zerofilling, was 1,024 . The voxel was placed in close proximity to the femur, limiting motion and avoiding EMCL and vasculature. Voxel-specific shimming was performed using the pencil beam technique to second order. IMCL peak area was normalized to the area of the water peak (IMCL/water).

\section{Analysis of MRS Data}

${ }^{1}$ H-MRS data were processed using LCModel software (Figure 1), which fits in vivo proton MR spectra to a linear combination of in vitro metabolite basis spectra (Provencher, 1993). LCModel returns residuals defining the difference between the acquired data and the modeled spectra, with larger residuals indicating inability of the model to accurately describe observed spectral amplitudes. After analysis, quality control criteria were implemented. These involved evaluating the baseline quality, residual amplitudes, and metabolite peak resolution within the acquired spectra.

A numerical value was assigned to each of these quality control measures as follows. Baseline quality was scored as 0 if a spectrum contained broad baseline components, and 1 if the baseline was sufficiently flat that it did not interfere with the measurement of the IMCL resonance amplitude. Residual amplitude was scored between 0 and 2, with 0 indicating differences $>15 \%$ of peak height, 1 indicating a difference between 5 and 15\% of the peak height, and 2 indicating a difference of $<5 \%$. Peak resolution was scored between 0 and 2, with 0 indicating complete lack of resolution of IMCL and EMCL peaks, 1 indicating a visible shoulder indicating the presence of two resonances, and 2 indicating peaks resolved down to $2 / 3$ of the IMCL peak amplitude or better. Many cases that received a score of zero for peak resolution had dominant EMCL resonances, rendering quantification of the IMCL peak unreliable. Only spectra with a score of 1 or more for each criterion were eligible for analysis. This dataset consisted of 938 spectra. Of these, 299 were removed according to the exclusion criteria. Of the remaining 639 spectra, 149 were longitudinal duplicates from the same participant. After removing these, the final MRS dataset consisted of data from 490 participants. Of these, 314 subjects had CT-based muscle density measurements of the thigh, and were therefore included in the analysis, with all of these subjects also having measurements of BMI, cholesterol, waist circumference, and circulating triglycerides.

\section{Computerized Tomography (CT)}

Muscle density was measured using computerized tomography (CT; Somatom Sensation 10; Siemens, Malvern, PA, United States) and quantified with BonAlyse software (Jyvaskyla, Finland). Tissue Identification and Quantification (TIDAQ) was used to generate tissue masks, which excluded intramuscular adipose tissue (IMAT). The accurately segmented compartments included air, fat, tendon, muscle, trabecular bone, and cortical bone (Makrogiannis et al., 2018). The mean density was calculated over all muscle included in the scanned slice of the mid-thigh (Figure 2). Each processed image was also visually evaluated, ensuring that there was no inaccurately identified tissue. Participants with low EMCL showed higher muscle quality and well-resolved muscle compartments when compared to participants with greater EMCL. Muscle attenuation values were reported in Hounsfield units (HU).

\section{Ancillary Variable Quantification}

Obesity status was quantified using body mass index (BMI), which was measured as weight $(\mathrm{kg}) /$ height $(\mathrm{m})^{2}$. Waist circumference was defined as the mean of upper abdominal circumference measurements $(\mathrm{cm})$. Serum cholesterol and triglyceride concentration were measured using a standard clinical lipid panel assay. Physical activity was self-reported via a questionnaire, with values ranging from zero (sedentary) to three (very active). Physical activity scores were reported by 312 participants.

\section{Statistical Analysis}

All statistical analyses were performed in RStudio version 1.2.1335. Data were reported as mean (standard deviation), and statistical significance was defined as $p<0.05$.

\section{Covariate Selection}

In preliminary analyses, trends in the data were summarized by locally weighted scatterplot smoothing (LOWESS) (Cleveland and Devlin, 1988). In an effort to evaluate the association between IMCL/water and muscle density independent of obesity, we investigated biomarkers that would plausibly correlate with BMI. These were BMI itself, serum cholesterol, circulating triglycerides and waist circumference (Bray et al., 2018). The effects of these potential confounders were evaluated by assessing individual correlations of IMCL/water with waist circumference, circulating triglyceride concentration, BMI and serum cholesterol levels. Confounders that were found to be significantly correlated with IMCL/water were included as covariates in the final 


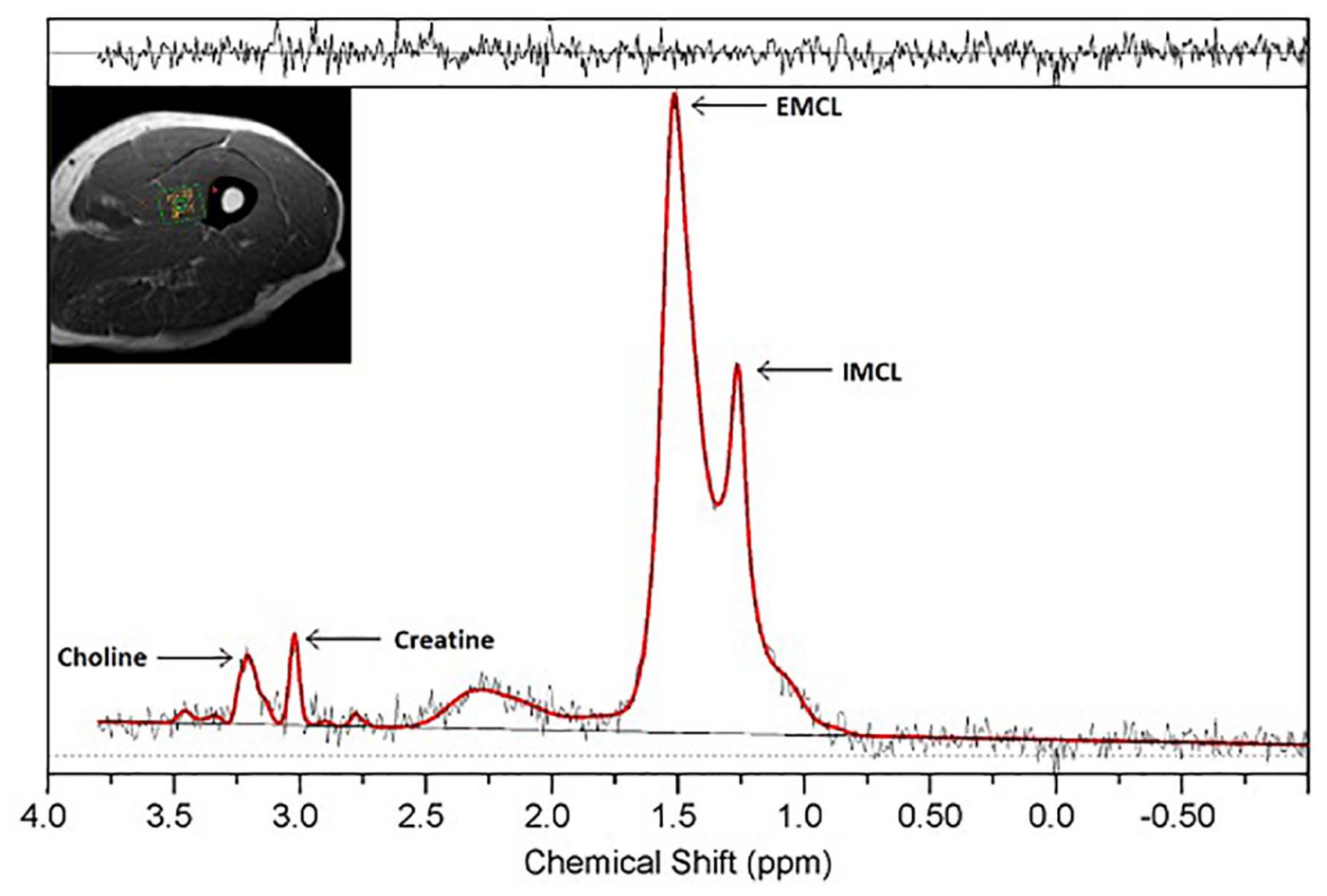

FIGURE 1 | ${ }^{1} \mathrm{H}-\mathrm{MRS}$ spectrum obtained from a single voxel in thigh muscle at 3.0 T. Resonances of IMCL (1.25 ppm), EMCL (1.4 ppm), choline (3.2 ppm), and creatine $(3.0 \mathrm{ppm})$ are well-resolved. Clear resolution between the $\mathrm{MMCL}$ and $\mathrm{EMCL}$ resonance peaks is seen, with residuals displayed above the spectrum.

A

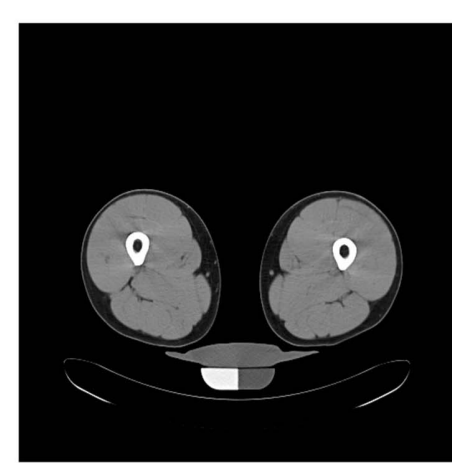

$\mathbf{B}$

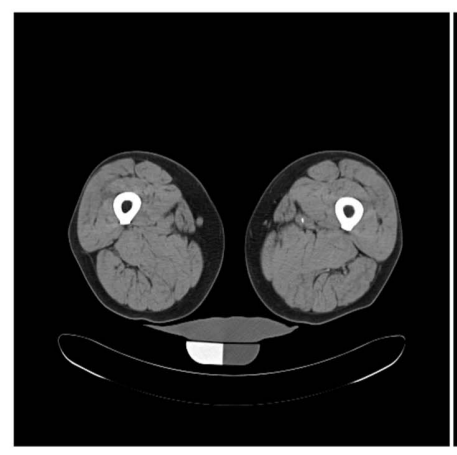

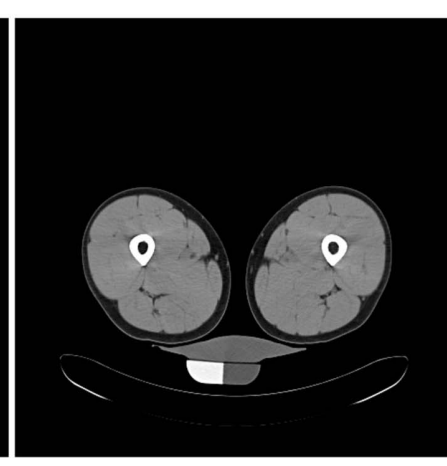
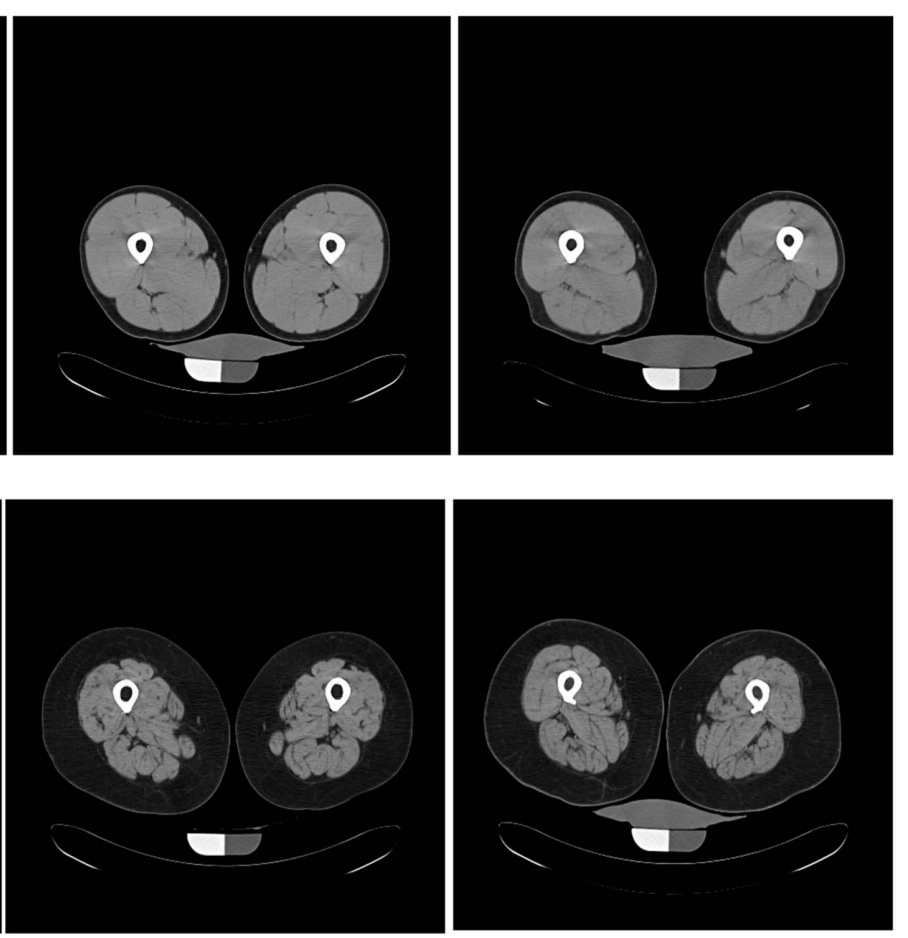

FIGURE 2 | Axial mid-thigh computerized tomography images acquired from three participants with (A) low EMCL measurements (0.0031 \pm 0.0002$)$ and three participants with $\mathbf{( B )}$ high EMCL measurements (0.202 \pm 0.085). Participants with low EMCL exhibit higher muscle CT density when compared to those with high EMCL content. 
TABLE 1 | Participant characteristics.

\begin{tabular}{|c|c|c|c|c|c|}
\hline & $n=314$ & Men $(n=142)$ & Women $(n=172)$ & Significance (Sex) & Significance (Race) \\
\hline Age (years) & $70.7 \pm 12.6$ & $71.4 \pm 12.6$ & $70.1 \pm 12.5$ & NS & $\mathrm{N} / \mathrm{A}$ \\
\hline BMI $\left(\mathrm{kg} / \mathrm{m}^{2}\right)$ & $26.5 \pm 4.0$ & $27.1 \pm 3.5$ & $25.9 \pm 4.3$ & $p<0.01$ & $p<0.001$ \\
\hline Waist circumference (cm) & $89.9 \pm 11.4$ & $97.1 \pm 8.6$ & $84.0 \pm 10.1$ & $p<0.001$ & $p<0.05$ \\
\hline Cholesterol (mg/dL) & $180.7 \pm 36.2$ & $168.4 \pm 32.8$ & $190.9 \pm 35.7$ & $p<0.001$ & NS \\
\hline Circulating triglyceride concentration (mg/dL) & $95.9 \pm 47.6$ & $103.1 \pm 56.0$ & $90.0 \pm 38.3$ & $p<0.05$ & $p<0.001$ \\
\hline Muscle attenuation (HU) & $50.5 \pm 3.3$ & $51.0 \pm 3.1$ & $50.0 \pm 3.4$ & $p<0.01$ & NS \\
\hline IMCL/water & $0.017 \pm 0.008$ & $0.019 \pm 0.008$ & $0.017 \pm 0.007$ & $p<0.05$ & NS \\
\hline Age adjusted IMCL/water & $0.018 \pm 0.008$ & $0.019 \pm 0.008$ & $0.017 \pm 0.007$ & $p<0.05$ & NS \\
\hline EMCL/water & $0.038 \pm 0.029$ & $0.033 \pm 0.031$ & $0.043 \pm 0.025$ & $p<0.001$ & NS \\
\hline
\end{tabular}

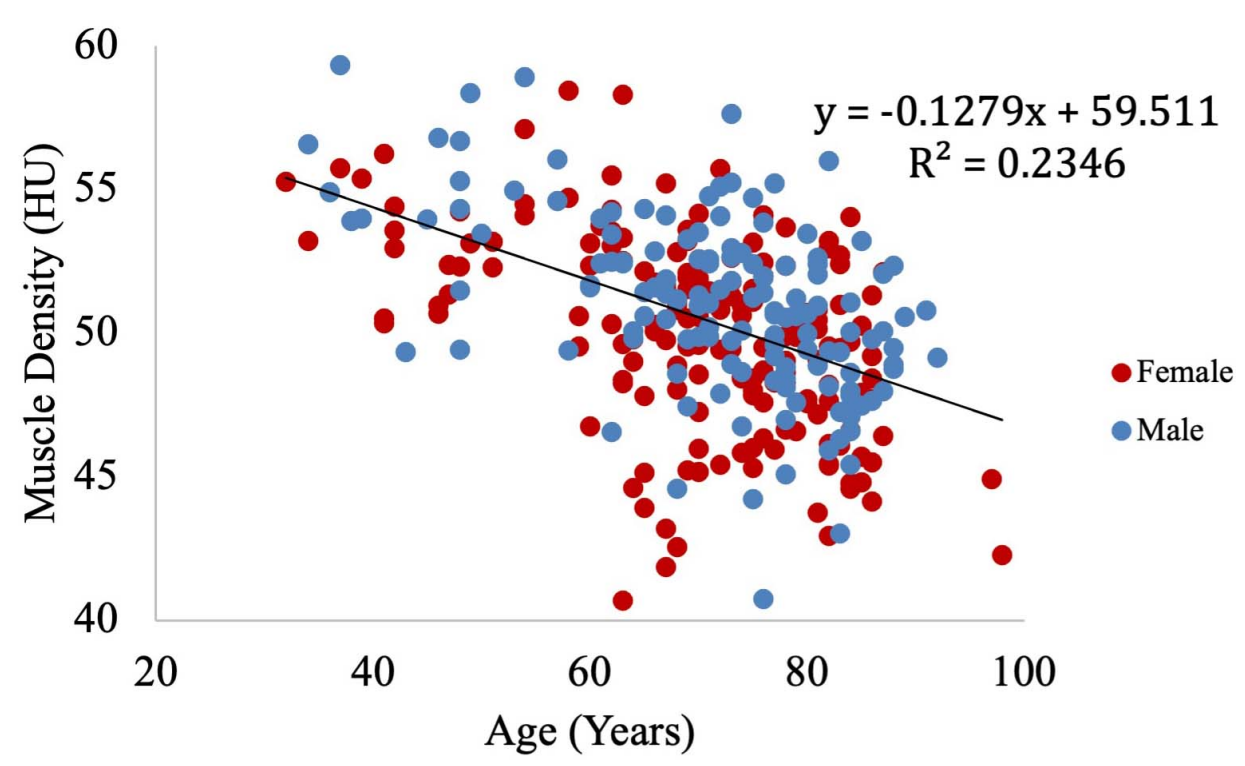

FIGURE 3 | Relationship between muscle density from CT and age. As expected, muscle density decreases significantly with age ( $p<0.001)$.

analysis; these were waist circumference, circulating triglyceride concentration and BMI.

\section{Age-Adjusted IMCL}

To examine the possible dependence on age of the correlation between IMCL/water and muscle density, a multivariate local polynomial regression (LOESS) analysis was performed to construct an age-adjusted IMCL variable. LOESS fits a model to a localized subset of data through multivariate smoothing and permits identification of independent variables responsible for the variation in a dependent variable.

\section{RESULTS}

The characteristics of the 314 participants (142 men, mean age 71.4 (12.6) years, age range 34-92 years; 172 women, mean age 70.1 (12.5) years, and age range 32-98 years) included in the analyses are reported in Table 1. Values are reflective of the good general health status of BLSA participants. The mean value of the IMCL resonance normalized by the water resonance was 0.017 (0.008), with a significant difference between men and women [women: 0.017 (0.007); men: 0.019 (0.008); $p=0.02$ ]. The mean value of the EMCL resonance normalized by the water resonance was $0.038(0.029)$, with a significant difference between men and women [women: 0.043 (0.025); men: 0.033 (0.031); $p<0.001]$. The average muscle attenuation in normalized Hounsfield units was 50.5 (3.3), with a significant difference found between women and men [women: 50.0 (3.4); men: 51.0 (3.1); $p<0.01]$.

Muscle density was evaluated as a function of age (Figure 3) and exhibited a significant inverse correlation $(p<0.001)$. A significant correlation was also found between muscle density and both IMCL/water (unadjusted; Figure 4) and EMCL/water (Figure 5). Of note, IMCL/water and age were not significantly associated (Figure 6). However, EMCL/water and age were significantly associated at $p<0.001$ (Figure 7).

Muscle density was then independently evaluated as a function of the obesity-related variables, including BMI (Figure 8), circulating triglyceride concentration (Figure 9), and waist circumference (Figure 10). After adjusting for IMCL/water, the significant relationship between muscle density and BMI became weaker. A similar effect was found in the 


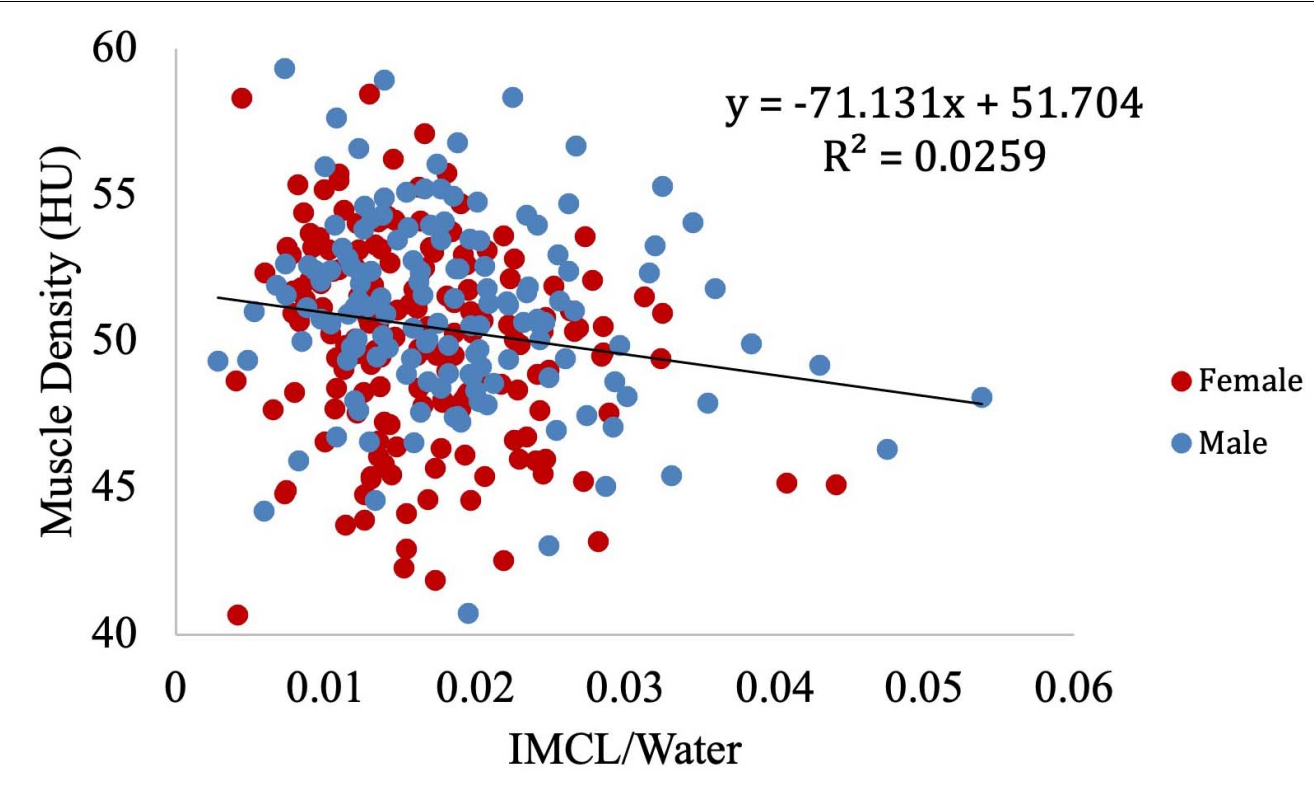

FIGURE 4 | Relationship between muscle density from CT and IMCL/water $(p<0.001)$.

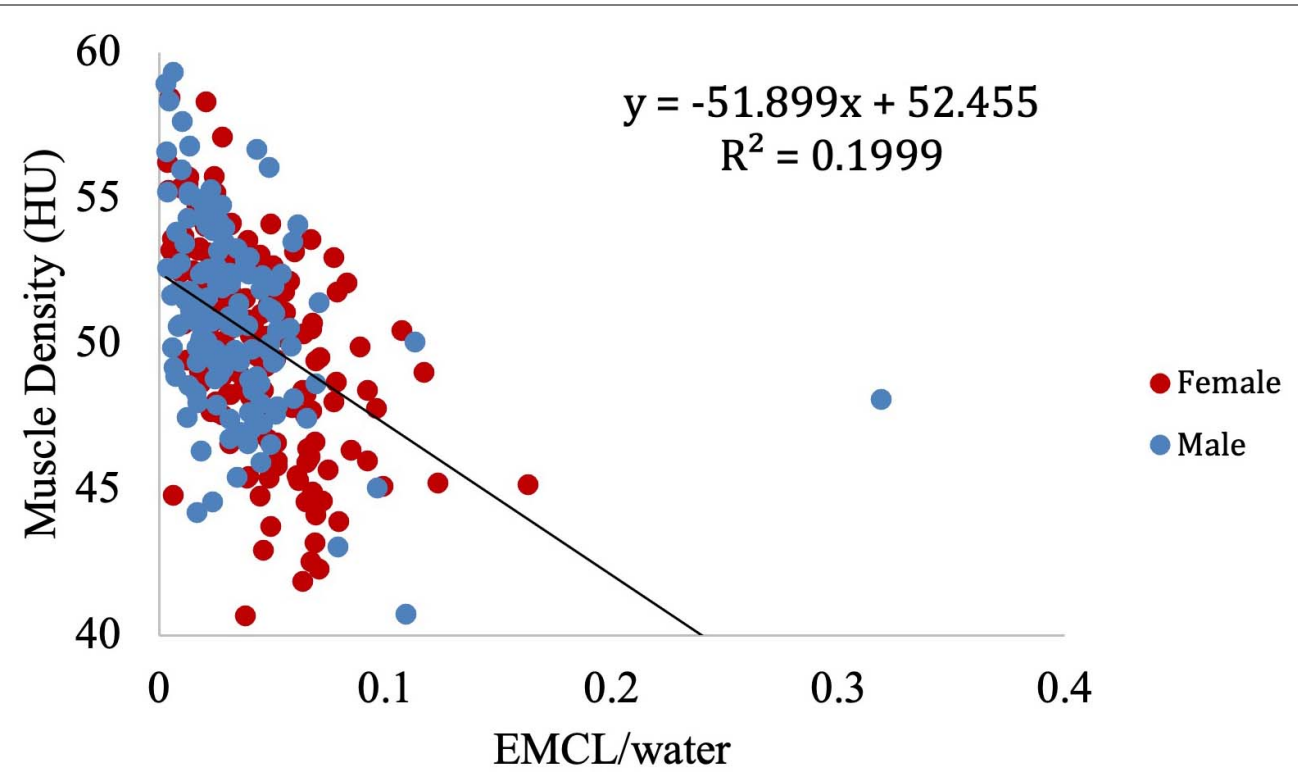

FIGURE 5 | Relationship between muscle density from CT and EMCL/water $(p<0.001)$.

muscle density and waist circumference model. However, adjusting for IMCL/water increased the significance of the relationship between muscle density and circulating triglyceride concentration.

In order to identify potential confounders of the muscle density and IMCL relationship, we examined clinical obesityrelated variables. Cholesterol was not significantly associated with IMCL/water and was excluded as a covariate, while BMI, waist circumference, and circulating triglyceride concentration were significantly correlated with IMCL/water and therefore included as covariates (Table 2).
After introducing age as a potential confounder in the regression model (Table 2: Model 1), the significant relationship between muscle density and IMCL/water was retained, and the inverse relationship between age and muscle density remained statistically significant. After further adjustment for physical activity, sex and race (Table 2: Model 2), the independent relationship between IMCL/water and muscle density remained statistically significant.

The role of obesity in the relationship between IMCL and muscle density was also investigated (Table 2: Model 3); with waist circumference being significantly associated with 


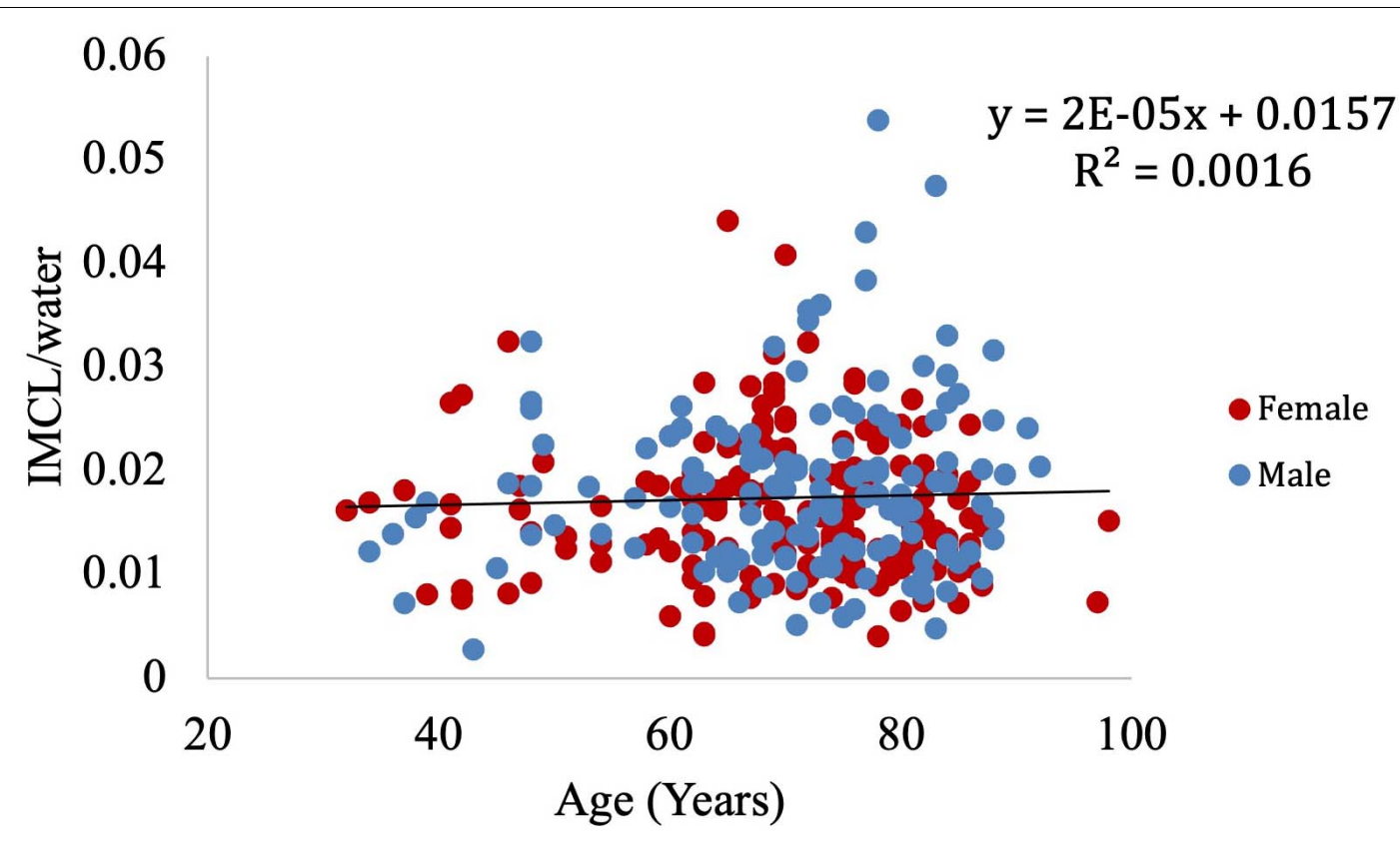

FIGURE 6 | Relationship between age and IMCL/water $(p=0.5)$.

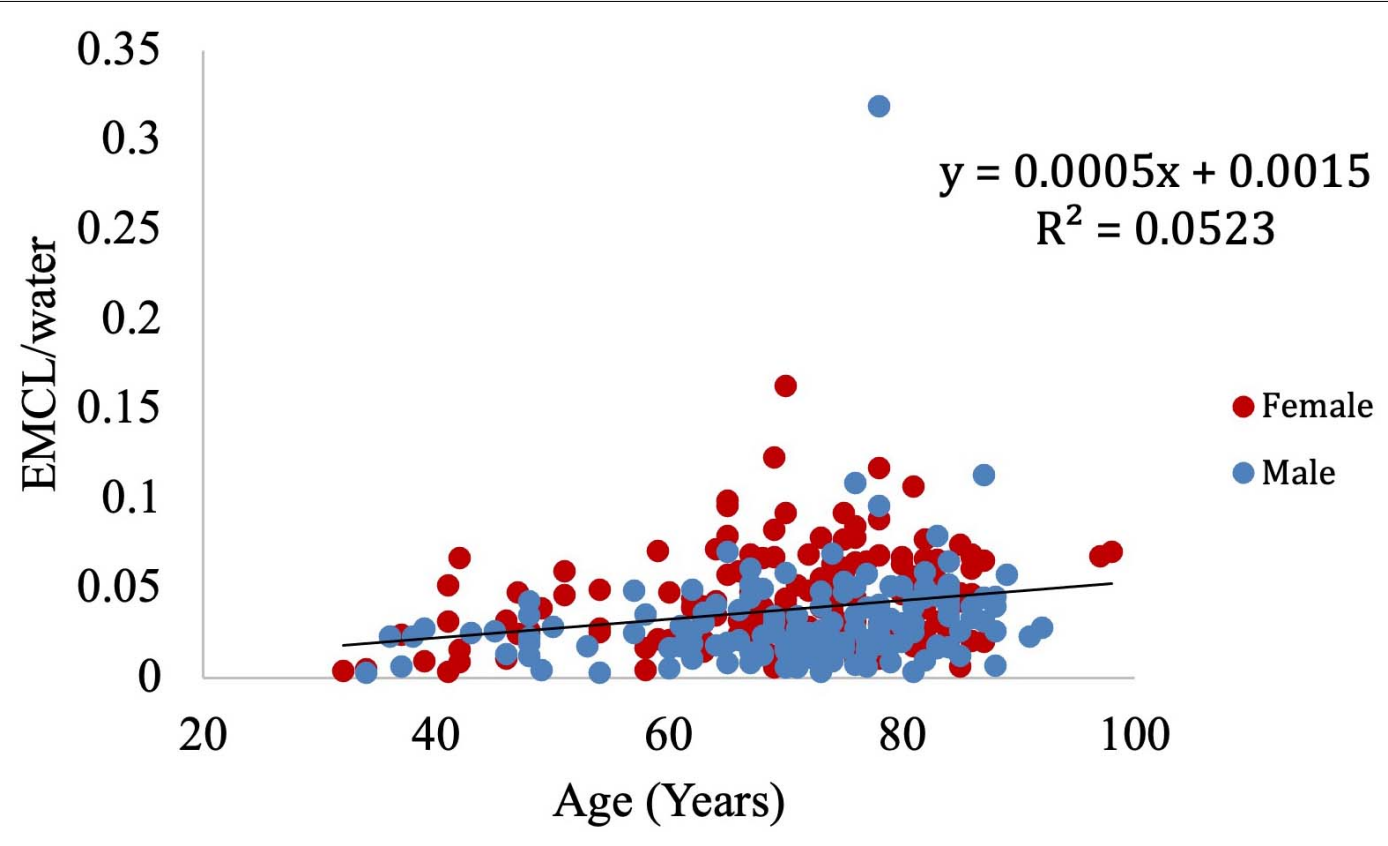

FIGURE 7 | Relationship between age and EMCL/water ( $p<0.001)$.

a lower muscle density. Surprisingly, circulating triglyceride concentration exhibited a significant positive correlation with muscle density. The analysis showed no significant interaction between CT values for muscle attenuation and BMI. After adjusting for age, sex, race, physical activity, BMI, circulating triglyceride concentration, and waist circumference, the relationship between IMCL/water and muscle density remained significant $(p<0.01)$.
To further visualize the relationship between IMCL and muscle density, LOESS regression was used to construct an age-adjusted IMCL/water variable. Muscle density was then evaluated as a function of age-adjusted IMCL/water (Figure 11). As in the non-adjusted analysis, greater age-adjusted IMCL/water was significantly associated with lower muscle density ( $p<0.01$; Table 3: Model 1). Additionally, circulating triglyceride concentration was found to be significantly and 


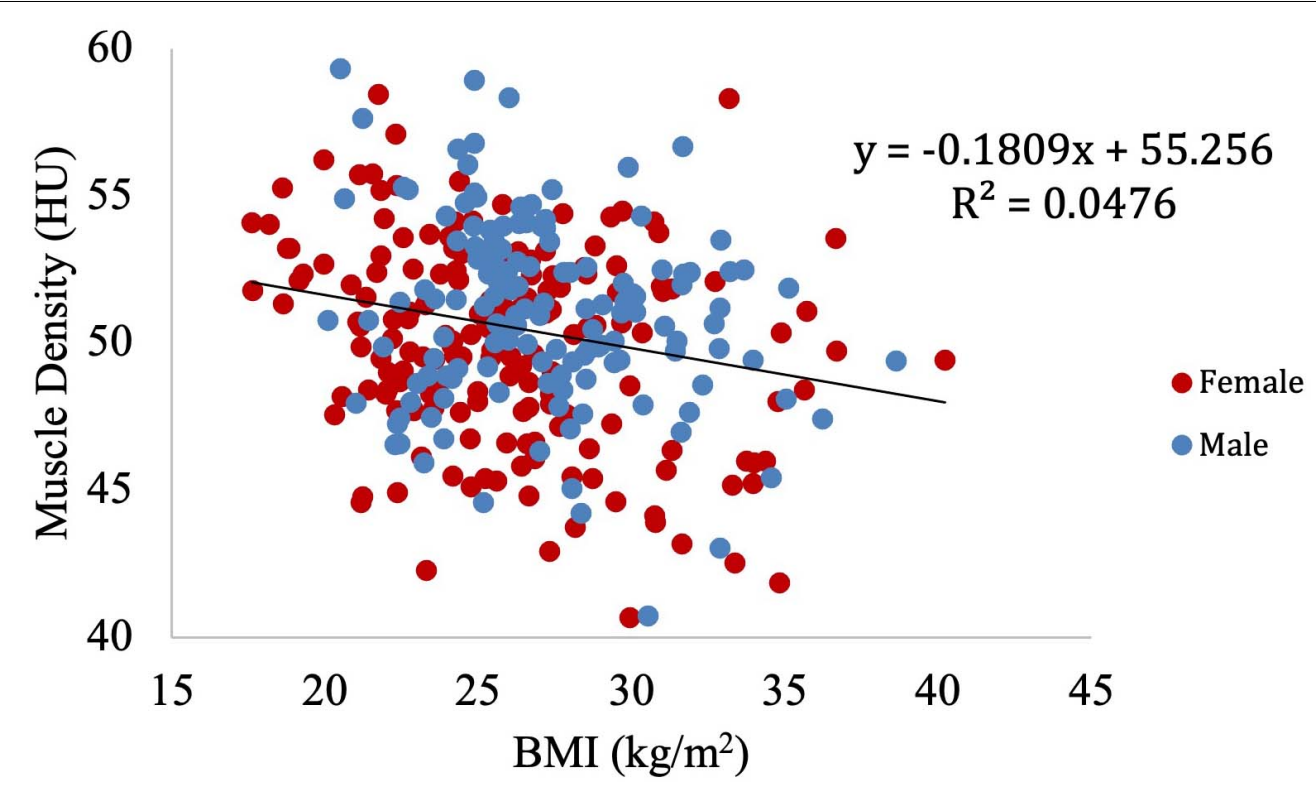

FIGURE 8 | Relationship between muscle density from CT and BMI $(p<0.01)$. This relationship remained significant $(p<0.01)$ after adjustment for IMCL/water.

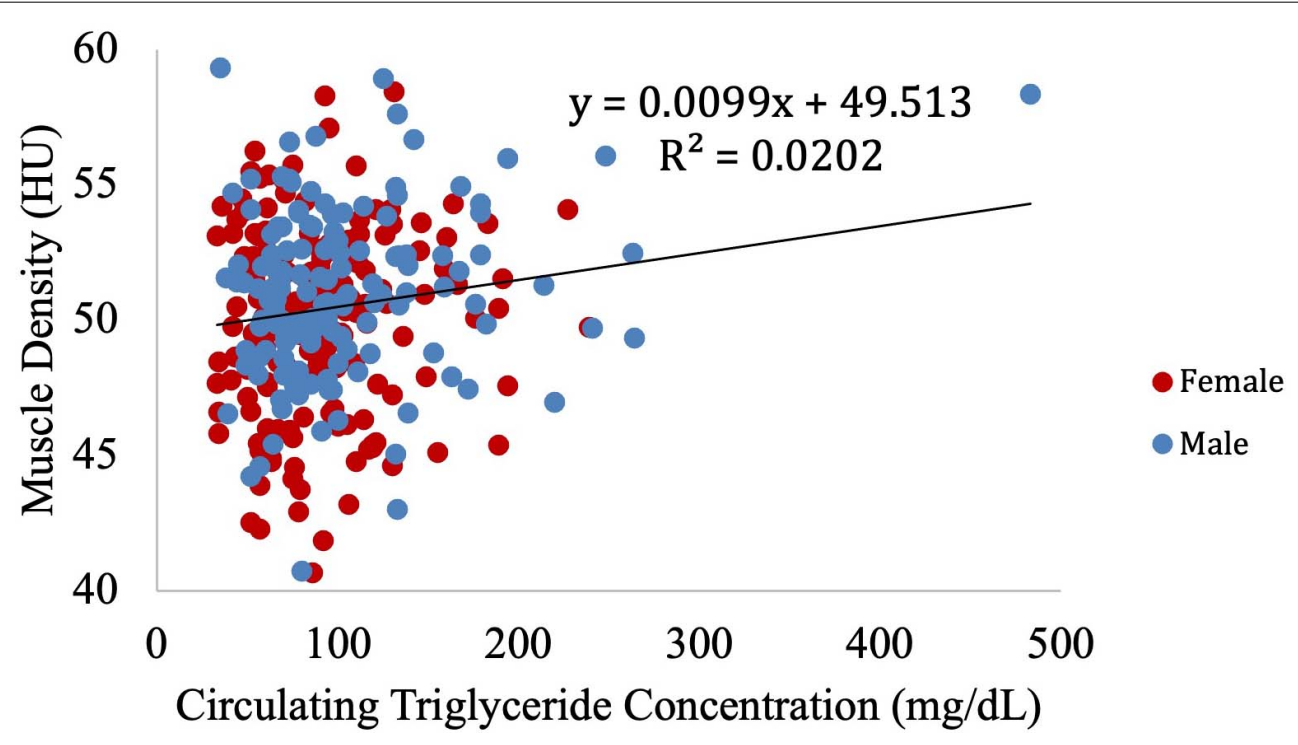

FIGURE 9 | Relationship between muscle density from CT and circulating triglyceride concentration $(p=0.18)$. After adjustment for IMCL/water, the relationship between muscle density and circulating triglyceride concentration became significant $(p=0.04)$. However, when the one participant with high triglyceride concentration is removed (circulating triglyceride value of 483), this correlation is no longer significant $(p=0.12)$.

positively correlated with muscle attenuation, while waist circumference showed a significant negative correlation. No significant correlation was found between muscle attenuation and BMI. After adjusting for all covariates mentioned above, the relationship between age-adjusted IMCL/water and muscle density remained significant $(p<0.05)$, suggesting that the association is independent of physical activity level, sex, race (Table 3: Model 2), and general obesity markers (Table 3: Model 3 ). The association was linear across the muscle attenuation range of 40.7-59.3 normalized Hounsfield units.

\section{DISCUSSION}

We found a statistically significant inverse relationship between IMCL/water ratio and muscle density. This relationship was maintained after formal adjustment for demographic and obesity-related variables. Our findings are consistent with those reported by Goodpaster et al. (2000a) in which muscle attenuation values from CT were negatively correlated with skeletal muscle lipid content, as determined by muscle biopsy followed by quantification of intramuscular fat with 


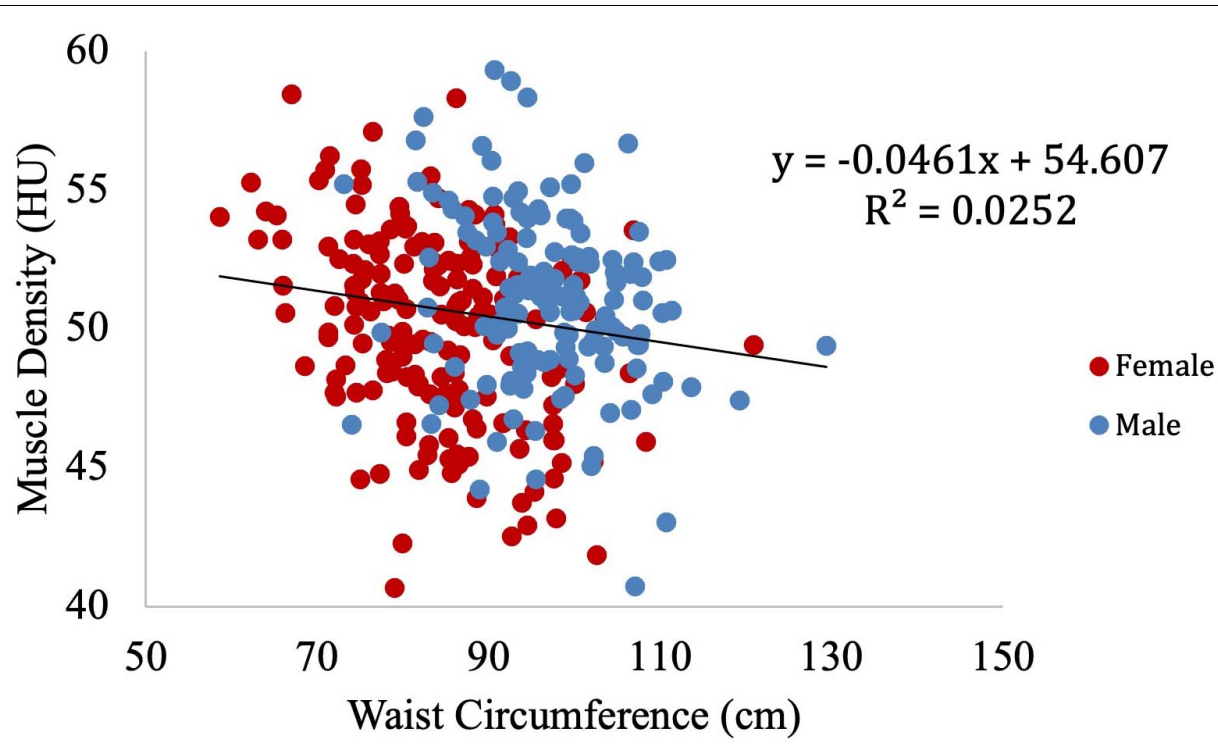

FIGURE $10 \mid$ Relationship between muscle density from CT and waist circumference $(p<0.01)$. This relationship remained significant $(p<0.01)$ after adjustment for IMCL/water.

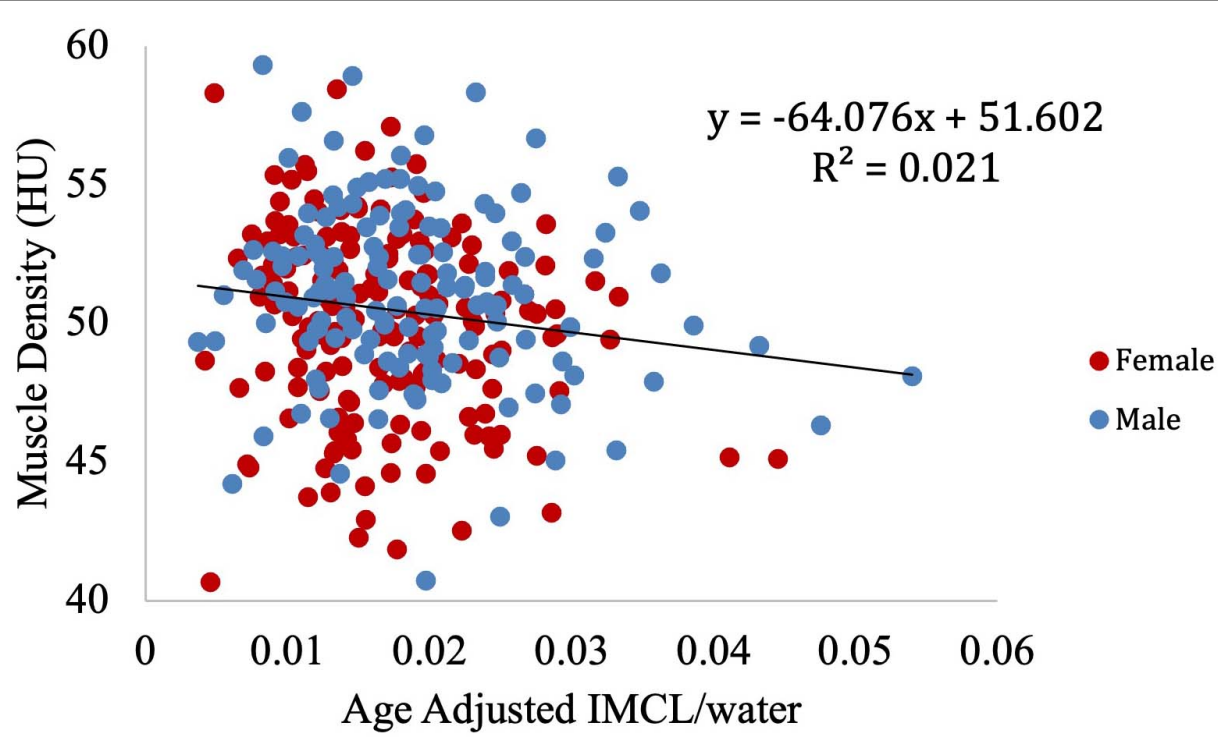

FIGURE 11 | Correlation between age-adjusted IMCL/water ratio obtained from MR spectroscopy and muscle density obtained from CT. As shown, decreased density was associated with increased age adjusted IMCL/water $(p<0.01)$. The age adjusted IMCL/water values were calculated using a LOESS regression.

histochemical analysis. While causation was not addressed in our work, the reported correlations suggest a hypothesis that the accumulation of IMCL may be a significant contributor to the reduction of muscle density with aging. This would form the basis for further investigation through a longitudinal study.

Aging is associated with decreased muscle strength, mass and density (Doherty, 2003; Newman et al., 2005; Kuk et al., 2009; Miljkovic and Zmuda, 2010). The decline in strength contributes to the diminished physical performance with aging (Brill et al., 2000). Given the established correlation between muscle density and strength, IMCL accumulation may contribute directly to the decrease in muscle strength and function with age (Siparsky et al., 2014), in addition to its likely causative role in metabolic disorders.

Our results are important because while muscle density is a crucial correlate of the disabling effect of aging and many chronic diseases and is associated with increased hospitalizations 
TABLE 2 | Multivariate analysis adjusting for confounding variables.

\begin{tabular}{|c|c|c|c|c|c|c|}
\hline \multirow[b]{2}{*}{ Parameters } & \multicolumn{2}{|c|}{ Model 1: MD = IMCL } & \multicolumn{2}{|c|}{ Model 2: MD = IMCL + Race + Sex } & \multicolumn{2}{|c|}{ Model 3: MD = IMCL + Race + Sex + Obesity } \\
\hline & $\beta(95 \% \mathrm{Cl})$ & $P$-value & $\beta(95 \% \mathrm{Cl})$ & $P$-value & $\beta(95 \% \mathrm{Cl})$ & $P$-value \\
\hline IMCL/water & $\begin{array}{l}-62.64 \\
(-84.28 \\
-41.00)\end{array}$ & $0.004^{\star \star}$ & $\begin{array}{c}-76.48(-97.70 \\
-55.26)\end{array}$ & $<0.001^{\star \star \star}$ & $-60.61(-81.75,-39.47)$ & $0.004^{\star \star}$ \\
\hline Age & $\begin{array}{l}-0.126 \\
(-0.139 \\
-0.113)\end{array}$ & $<0.001^{\star \star \star}$ & $\begin{array}{c}-0.124(-0.136 \\
-0.111)\end{array}$ & $<0.001^{\star \star \star}$ & $-0.120(-0.132,-0.108)$ & $<0.001^{\star \star \star}$ \\
\hline Race & - & - & $0.248(0.097,0.399)$ & 0.101 & $-0.019(-0.171,0.133)$ & 0.899 \\
\hline Sex & - & - & $1.19(0.861,1.52)$ & $<0.001^{\star \star \star}$ & $1.92(1.48,2.36)$ & $<0.001^{\star \star \star}$ \\
\hline Physical activity & - & - & $0.390(0.209,0.571)$ & $<0.05^{\star}$ & $0.278(0.103,0.453)$ & 0.113 \\
\hline $\mathrm{BMI}\left(\mathrm{kg} / \mathrm{m}^{2}\right)$ & - & - & - & - & $-0.082(-0.152,-0.012)$ & 0.238 \\
\hline $\begin{array}{l}\text { Waist } \\
\text { Circumference (cm) }\end{array}$ & - & - & - & - & $-0.061(-0.091,-0.031)$ & $0.041^{*}$ \\
\hline $\begin{array}{l}\text { Circulating } \\
\text { triglyceride } \\
\text { concentration } \\
(\mathrm{mg} / \mathrm{dL})\end{array}$ & - & - & - & - & $0.012(0.009,0.015)$ & $<0.001^{* *}$ \\
\hline
\end{tabular}

IMCL/water is a significant covariate in the relationship between muscle density (MD) and age, indicating that increased IMCL may play a significant role in this relationship. ${ }^{* * *} p<0.001,{ }^{* *} p<0.01$, and ${ }^{*} p<0.05$. Physical activity data were available for 312 participants.

TABLE 3 | Multivariate analysis adjusting for confounding variables.

\begin{tabular}{|c|c|c|c|c|c|c|}
\hline \multirow[b]{2}{*}{ Parameters } & \multicolumn{2}{|c|}{ Model 1: MD = Age Adjusted IMCL } & \multicolumn{2}{|c|}{$\begin{array}{c}\text { Model 2: MD = Age Adjusted } \\
\text { IMCL + Race + Sex }\end{array}$} & \multicolumn{2}{|c|}{$\begin{array}{c}\text { Model 3: MD }=\text { Age Adjusted IMCL + } \\
\text { Race }+ \text { Sex + Obesity }\end{array}$} \\
\hline & $\beta(95 \% \mathrm{Cl})$ & $P$-value & $\beta(95 \% \mathrm{Cl})$ & $P$-value & $\beta(95 \% \mathrm{Cl})$ & $P$-value \\
\hline $\begin{array}{l}\text { Age adjusted } \\
\text { IMCL/water }\end{array}$ & $\begin{array}{c}-64.08(-88.84 \\
-39.32)\end{array}$ & $0.010^{*}$ & $\begin{array}{l}-75.79(-100.13 \\
-51.45)\end{array}$ & $<0.01^{\star \star}$ & $-60.11(-84.36,-35.86)$ & $0.014^{*}$ \\
\hline Race & - & - & $0.263(0.090,0.436)$ & 0.123 & $-0.041(-0.216,0.134)$ & 0.816 \\
\hline Sex & - & - & $0.914(0.539,1.29)$ & $<0.05^{\star}$ & $2.13(1.63,2.63)$ & $<0.001^{\star \star \star}$ \\
\hline Physical activity & - & - & $0.664(0.459,0.869)$ & $<0.01^{\star \star}$ & $0.551(0.353,0.749)$ & $<0.01^{\star \star}$ \\
\hline BMl & - & - & - & - & $0.032(-0.047,0.111)$ & 0.690 \\
\hline $\begin{array}{l}\text { Waist } \\
\text { circumference }(\mathrm{cm})\end{array}$ & - & - & - & - & $-0.111(-0.145,-0.077)$ & $<0.001^{* * *}$ \\
\hline $\begin{array}{l}\text { Circulating } \\
\text { triglyceride } \\
\text { concentration } \\
(\mathrm{mg} / \mathrm{dL})\end{array}$ & - & - & - & - & $0.015(0.011,0.019)$ & $<0.001^{\star * *}$ \\
\hline
\end{tabular}

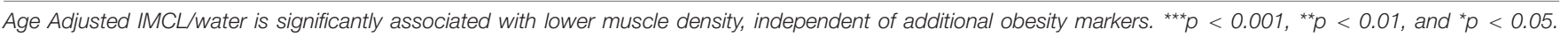
Physical activity data were available for 312 participants.

in older persons, the mechanistic underpinnings of decreased muscle density remain elusive (Cawthon et al., 2009; Delmonico et al., 2009; Demontis et al., 2013). Aside from its role in aging, low muscle density has been shown to correlate with other pathology, including rheumatoid arthritis (RA), a systemic inflammatory condition that can result in disability and joint deformity. In particular, CT-determined thigh muscle density was found to correlate with increased serum interleukin-6 levels, glucocorticoid requirement, number of joints exhibiting tenderness, and disease progression (Kramer et al., 2012). Muscle density is also associated with physical function in idiopathic inflammatory myopathy (IIM) patients. In these individuals, low muscle density is correlated with a decrease in physical function, endurance, and strength (Cleary et al., 2015). In these studies, however, the potential role of fat infiltration as a causative factor in lower muscle density was not investigated. Low muscle density is also considered a biomarker of impaired metabolism and it is often observed in people with increased insulin resistance and metabolic syndrome (Kim et al., 2003). Similarly, increased levels of IMCL are associated with insulin resistance in obese patients (Pan et al., 1997). Insulin resistance in muscle is in part characterized by decreased oxidative capacity and impaired fatty acid oxidation (Goodpaster et al., 2001b). Our findings reinforce the hypothesis that IMCL accumulation in skeletal myocytes is an essential part of the reduction of muscle density with aging and not merely the result of the confounding effects of body composition changes. Whether interventions that diminish IMCL infiltration also affect muscle quality, improve metabolic 
control and reduce insulin resistance remains an important topic for future studies.

We note that EMCL is expected to dominate IMCL in terms of overall fat content, even when macroscopic fat deposits are excluded. Accordingly, CT-based muscle density would depend more strongly on EMCL. However, as outlined above, EMCL, being much less readily accessible to intracellular machinery, is not as metabolically active as IMCL. Thus, in spite of its weaker correlation with HU, it is IMCL in which we are primarily interested. We have found this correlation to be operative on a population-based scale, though not on the scale of an individual subject.

Although, as far as we know, this is the largest study to date using ${ }^{1} \mathrm{H}$-MRS to quantify IMCL in muscle, certain limitations remain. Accurate quantification of IMCL by ${ }^{1} \mathrm{H}$-MRS requires strict attention to spectral quality. We ensured the accurate measurement of IMCL using ${ }^{1} \mathrm{H}$-MRS through application of specific quality control criteria. The most common reason for excluding a spectrum from our analysis was an absence of clear resolution between the IMCL and EMCL peaks, in which case IMCL cannot be accurately quantified. This lack of resolution was most often due to a large EMCL resonance from the selected spectral voxel, indicating high EMCL content. However, rejection of these spectra may have also excluded those with high IMCL concentrations, limiting our range in IMCL/water ratio. Nevertheless, this range is comparable to those found in the literature. Additionally, the presence of macroscopic, "marbled" fat can result in poorly resolved separation between IMCL and EMCL peaks. Removing these spectra from analysis has the potential to introduce bias through exclusion of individuals with a greater degree of obesity. Indeed, BMI data were available for 297 participants for which spectra were excluded. The average BMI of these participants was $27.4 \pm 3.9$, compared to an average BMI of $26.5 \pm 4.0$ for included participants. While this difference in BMI did reach statistical significance $(p<0.01)$, the difference in absolute terms was small $(\sim 3 \%)$.

Since ${ }^{1} \mathrm{H}$-MRS only provides normalized metabolite concentrations, we were unable to determine absolute water concentrations. However, previous work has indicated that the ratio IMCL/water was significantly correlated with IMCL normalized to an external oil phantom (Larson-Meyer et al., 2006). This supports the use of quantitative IMCL/water measurements in our analysis.

Voxel placement considerations represent a further limitation of our study. While the majority of participants had IMCL data obtained from the vastus medialis region of the lower extremity, data were collected from regions closer to the vastus intermedius for participants with large amounts of EMCL, as the vastus medialis was too small to contain the voxel without encroaching on macroscopic fat deposits. In these individuals with greater macroscopic fat, there was particularly poor resolution between these two muscles, making it very difficult to specify which provided the predominant contribution to the MRS signal. In addition, the CT imaging voxels and the corresponding MRI spectroscopic voxels could be co-localized, but not precisely registered. Briefly, the MRS technique used is a localizing technique, but not an imaging technique. The much larger MRS voxels will exhibit partial overlap with several CT pixels, and will not be registered with them, so there will not be a simple correspondence between the contents of the MRS voxel and a set of CT pixels. Moreover, the CT slice of $10 \mathrm{~mm}$ was considerably thinner than the MRS voxel, which was up to $40 \mathrm{~mm}$ long in the inferior-superior direction, depending on voxel orientation. Thus, the MRS values will include contributions from regions not covered by CT. Nevertheless, while we cannot register on a pixelby-pixel basis, we did obtain data from the same region using the two techniques to achieve the desired correspondence.

Use of the BLSA population enabled us to perform measurements in a relatively well-controlled healthy cohort across a large age range but may limit the applicability of these results to more typical populations. Nevertheless, our methods permitted an assessment of the correlation between IMCL and the decrease in muscle density with age, which is expected to be generalizable. The present correlative findings support further investigation of the hypothesis that increased concentration of IMCL is associated with poor muscle quality; our results are consistent with but greatly extend previous findings (Larson-Meyer et al., 2006).

In sum, we find that the correlation between IMCL and functional biomarkers, and in particular muscle density assessed through CT, cannot be meaningfully established in an individual subject. However, population-based IMCL measurement may serve as an indicator of metabolic and functional health in the aging population.

\section{DATA AVAILABILITY STATEMENT}

The raw data supporting the conclusions of this article will be made available by the authors, without undue reservation.

\section{ETHICS STATEMENT}

The studies involving human participants were reviewed and approved by the Ethics Branch, National Institute on Aging/NIH, Bethesda, MD. The patients/participants provided their written informed consent to participate in this study.

\section{AUTHOR CONTRIBUTIONS}

$\mathrm{NB}, \mathrm{KF}, \mathrm{DR}, \mathrm{RS}$, and LF conceived the study. NB, RS, and LF analyzed the data gathered, performed statistical analysis, and drafted the manuscript. All the authors contributed to the manuscript editing, and read and approved the submitted version.

\section{ACKNOWLEDGMENTS}

This work was supported by the Intramural Research Program of the NIH, National Institute on Aging. We would like to thank a reviewers for particularly helpful comments. 


\section{REFERENCES}

Addison, O., Marcus, R. L., Lastayo, P. C., and Ryan, A. S. (2014). Intermuscular fat: a review of the consequences and causes. Int. J. Endocrinol. 2014:309570. doi: 10.1155/2014/309570

Aguer, C., Mercier, J., Man, C. Y., Metz, L., Bordenave, S., Lambert, K., et al. (2010). Intramyocellular lipid accumulation is associated with permanent relocation ex vivo and in vitro of fatty acid translocase (FAT)/CD36 in obese patients. Diabetologia 53, 1151-1163. doi: 10.1007/s00125-010-1708- $\mathrm{x}$

Akil, L., and Ahmad, H. A. (2011). Relationships between obesity and cardiovascular diseases in four southern states and Colorado. J. Health Care Poor Underserved 22(4 Suppl), 61-72. doi: 10.1353/hpu.2011.0166

Amin, F., Fatima, S. S., Islam, N., and Gilani, A. H. (2015). Prevalence of obesity and overweight, its clinical markers and associated factors in a high risk South-Asian population. BMC Obes. 2:16. doi: 10.1186/s40608-015-0044-6

Anderson, D. E., D’Agostino, J. M., Bruno, A. G., Demissie, S., Kiel, D. P., and Bouxsein, M. L. (2013). Variations of CT-based trunk muscle attenuation by age, sex, and specific muscle. J. Gerontol. A Biol. Sci. Med. Sci. 68, 317-323. doi: 10.1093 /gerona/gls168

Aubrey, J., Esfandiari, N., Baracos, V. E., Buteau, F. A., Frenette, J., Putman, C. T. et al. (2014). Measurement of skeletal muscle radiation attenuation and basis of its biological variation. Acta Physiol. (Oxf.) 210, 489-497. doi: 10.1111/apha. 12224

Boesch, C. (2007). Musculoskeletal spectroscopy. J. Magn. Reson. Imaging 25, 321-338. doi: 10.1002/jmri.20806

Boesch, C., Slotboom, J., Hoppeler, H., and Kreis, R. (1997). In vivo determination of intra-myocellular lipids in human muscle by means of localized $1 \mathrm{H}-\mathrm{MR}$ spectroscopy. Magn. Reson. Med. 37, 484-493. doi: 10.1002/mrm.1910370403

Bray, G. A., Heisel, W. E., Afshin, A., Jensen, M. D., Dietz, W. H., Long, M., et al. (2018). The science of obesity management: an Endocrine Society scientific statement. Endocr. Rev. 39, 79-132. doi: 10.1210/er.2017-00253

Bredella, M. A., Gill, C. M., Gerweck, A. V., Landa, M. G., Kumar, V., Daley, S. M., et al. (2013). Ectopic and serum lipid levels are positively associated with bone marrow fat in obesity. Radiology 269, 534-541. doi: 10.1148/radiol.13130375

Brill, P. A., Macera, C. A., Davis, D. R., Blair, S. N., and Gordon, N. (2000). Muscular strength and physical function. Med. Sci. Sports Exerc. 32, 412-416. doi: 10.1097/00005768-200002000-00023

Brumbaugh, D. E., Crume, T. L., Nadeau, K., Scherzinger, A., and Dabelea, D. (2012). Intramyocellular lipid is associated with visceral adiposity, markers of insulin resistance, and cardiovascular risk in prepubertal children: the EPOCH study. J. Clin. Endocrinol. Metab. 97, E1099-E1105. doi: 10.1210/jc.2011-3243

Cawthon, P. M., Fox, K. M., Gandra, S. R., Delmonico, M. J., Chiou, C. F., Anthony, M. S., et al. (2009). Do muscle mass, muscle density, strength, and physical function similarly influence risk of hospitalization in older adults? J. Am. Geriatr. Soc. 57, 1411-1419. doi: 10.1111/j.1532-5415.2009.02366.x

Cesari, M., Leeuwenburgh, C., Lauretani, F., Onder, G., Bandinelli, S., Maraldi, C., et al. (2006). Frailty syndrome and skeletal muscle: results from the Invecchiare in Chianti study. Am. J. Clin. Nutr. 83, 1142-1148. doi: 10.1093/ajcn/83.5.1142

Choi, S. J., Files, D. C., Zhang, T., Wang, Z. M., Messi, M. L., Gregory, H., et al. (2016). Intramyocellular lipid and impaired myofiber contraction in normal weight and obese older adults. J. Gerontol. A Biol. Sci. Med. Sci. 71, 557-564. doi: 10.1093/gerona/glv169

Cleary, L. C., Crofford, L. J., Long, D., Charnigo, R., Clasey, J., Beaman, F., et al. (2015). Does computed tomography-based muscle density predict muscle function and health-related quality of life in patients with idiopathic inflammatory myopathies? Arthritis Care Res. (Hoboken) 67, 1031-1040. doi: 10.1002/acr.22557

Cleveland, W. S., and Devlin, S. J. (1988). Locally weighted regression: an approach to regression analysis by local fitting. J. Am. Stat. Assoc. 83, 596-610. doi: 10.1080/01621459.1988.10478639

Conroy, M. B., Kwoh, C. K., Krishnan, E., Nevitt, M. C., Boudreau, R., Carbone, L. D., et al. (2012). Muscle strength, mass, and quality in older men and women with knee osteoarthritis. Arthritis Care Res. (Hoboken) 64, 15-21. doi: 10.1002/ acr.20588

Cox, R. A., and Garcia-Palmieri, M. R. (1990). "Cholesterol, triglycerides, and associated lipoproteins," in Clinical Methods: The History, Physical, and Laboratory Examinations, eds H. K. Walker, W. D. Hall, and J. W. Hurst (Boston, MA: Butterworths).
Cree, M. G., Newcomer, B. R., Katsanos, C. S., Sheffield-Moore, M., Chinkes, D., Aarsland, A., et al. (2004). Intramuscular and liver triglycerides are increased in the elderly. J. Clin. Endocrinol. Metab. 89, 3864-3871. doi: 10.1210/jc.2003031986

Delmonico, M. J., Harris, T. B., Visser, M., Park, S. W., Conroy, M. B., VelasquezMieyer, P., et al. (2009). Longitudinal study of muscle strength, quality, and adipose tissue infiltration. Am. J. Clin. Nutr. 90, 1579-1585. doi: 10.3945/ajcn. 2009.28047

Demontis, F., Piccirillo, R., Goldberg, A. L., and Perrimon, N. (2013). Mechanisms of skeletal muscle aging: insights from Drosophila and mammalian models. Dis. Model. Mech. 6, 1339-1352. doi: 10.1242/dmm.012559

Doherty, T. J. (2003). Invited review: aging and sarcopenia. J. Appl. Physiol. (1985) 95, 1717-1727. doi: 10.1152/japplphysiol.00347.2003

Ferrucci, L. (2008). The Baltimore Longitudinal Study of Aging (BLSA): a 50-yearlong journey and plans for the future. J. Gerontol. A Biol. Sci. Med. Sci. 63, 1416-1419. doi: 10.1093/gerona/63.12.1416

Golay, A., and Ybarra, J. (2005). Link between obesity and type 2 diabetes. Best Pract. Res. Clin. Endocrinol. Metab. 19, 649-663. doi: 10.1016/j.beem.2005. 07.010

Goodpaster, B. H., Carlson, C. L., Visser, M., Kelley, D. E., Scherzinger, A., Harris, T. B., et al. (2001a). Attenuation of skeletal muscle and strength in the elderly: the Health ABC study. J. Appl. Physiol. (1985) 90, 2157-2165. doi: 10.1152/ jappl.2001.90.6.2157

Goodpaster, B. H., He, J., Watkins, S., and Kelley, D. E. (2001b). Skeletal muscle lipid content and insulin resistance: evidence for a paradox in endurancetrained athletes. J. Clin. Endocrinol. Metab. 86, 5755-5761. doi: 10.1210/jcem. 86.12.8075

Goodpaster, B. H., Kelley, D. E., Thaete, F. L., He, J., and Ross, R. (2000a). Skeletal muscle attenuation determined by computed tomography is associated with skeletal muscle lipid content. J. Appl. Physiol (1985) 89, 104-110. doi: 10.1152/ jappl.2000.89.1.104

Goodpaster, B. H., Theriault, R., Watkins, S. C., and Kelley, D. E. (2000b) Intramuscular lipid content is increased in obesity and decreased by weight loss. Metabolism 49, 467-472. doi: 10.1016/S0026-0495(00)80010-4

Hicks, G. E., Simonsick, E. M., Harris, T. B., Newman, A. B., Weiner, D. K., Nevitt, M. A., et al. (2005). Cross-sectional associations between trunk muscle composition, back pain, and physical function in the Health, Aging and Body Composition study. J. Gerontol. A Biol. Sci. Med. Sci. 60, 882-887. doi: 10.1093/ gerona/60.7.882

Howald, H., Boesch, C., Kreis, R., Matter, S., Billeter, R., Essen-Gustavsson, B., et al. (2002). Content of intramyocellular lipids derived by electron microscopy, biochemical assays, and (1)H-MR spectroscopy. J. Appl. Physiol. (1985) 92, 2264-2272. doi: 10.1152/japplphysiol.01174.2001

Hwang, J. H., Pan, J. W., Heydari, S., Hetherington, H. P., and Stein, D. T. (2001) Regional differences in intramyocellular lipids in humans observed by in vivo 1H-MR spectroscopic imaging. J. Appl. Physiol. (1985) 90, 1267-1274. doi: 10.1152/jappl.2001.90.4.1267

Ingram, K. H., Lara-Castro, C., Gower, B. A., Makowsky, R., Allison, D. B., Newcomer, B. R., et al. (2011). Intramyocellular lipid and insulin resistance: differential relationships in European and African Americans. Obesity (Silver Spring) 19, 1469-1475. doi: 10.1038/oby.2011.45

Jacob, S., Machann, J., Rett, K., Brechtel, K., Volk, A., Renn, W., et al. (1999). Association of increased intramyocellular lipid content with insulin resistance in lean nondiabetic offspring of type 2 diabetic subjects. Diabetes 48, 1113-1119. doi: $10.2337 /$ diabetes.48.5.1113

Kalyani, R. R., Corriere, M., and Ferrucci, L. (2014). Age-related and disease-related muscle loss: the effect of diabetes, obesity, and other diseases. Lancet Diabetes Endocrinol. 2, 819-829. doi: 10.1016/S2213-8587(14)70034-8

Kim, D., Nam, S., Ahn, C., Kim, K., Yoon, S., Kim, J., et al. (2003). Correlation between midthigh low-density muscle and insulin resistance in obese nondiabetic patients in Korea. Diabetes Care 26, 1825-1830. doi: 10.2337/ diacare.26.6.1825

Kramer, H. R., Fontaine, K. R., Bathon, J. M., and Giles, J. T. (2012) Muscle density in rheumatoid arthritis: associations with disease features and functional outcomes. Arthritis Rheum. 64, 2438-2450. doi: 10.1002/art.3 4464

Krssak, M., Falk Petersen, K., Dresner, A., DiPietro, L., Vogel, S. M., Rothman, D. L., et al. (1999). Intramyocellular lipid concentrations are correlated with 
insulin sensitivity in humans: a 1H NMR spectroscopy study. Diabetologia 42, 113-116. doi: $10.1007 / \mathrm{s} 001250051123$

Kuk, J. L., Saunders, T. J., Davidson, L. E., and Ross, R. (2009). Age-related changes in total and regional fat distribution. Ageing Res. Rev. 8, 339-348. doi: 10.1016/j.arr.2009.06.001

Kurata, M., Narikawa, T., Waki, M., Koh, H., Maruyama, T., and Nambu, S. (1990). Relationships between serum cholesterol and obesity: a field study on nutritional background of hypercholesterolemia. Diabetes Res. Clin. Pract. 10(Suppl. 1), S239-S245. doi: 10.1016/0168-8227(90)90170-X

Lang, T., Cauley, J. A., Tylavsky, F., Bauer, D., Cummings, S., Harris, T. B., et al. (2010). Computed tomographic measurements of thigh muscle cross-sectional area and attenuation coefficient predict hip fracture: the Health, Aging, and Body Composition study. J. Bone Miner. Res. 25, 513-519. doi: 10.1359/jbmr. 090807

Larson-Meyer, D. E., Newcomer, B. R., and Hunter, G. R. (2002). Influence of endurance running and recovery diet on intramyocellular lipid content in women: a 1H NMR study. Am. J. Physiol. Endocrinol. Metab. 282, E95-E106. doi: 10.1152/ajpendo.2002.282.1.E95

Larson-Meyer, D. E., Smith, S. R., Heilbronn, L. K., Kelley, D. E., Ravussin, E., Newcomer, B. R., et al. (2006). Muscle-associated triglyceride measured by computed tomography and magnetic resonance spectroscopy. Obesity (Silver Spring) 14, 73-87. doi: 10.1038/oby.2006.10

Lauretani, F., Bandinelli, S., Bartali, B., Di Iorio, A., Giacomini, V., Corsi, A. M., et al. (2006). Axonal degeneration affects muscle density in older men and women. Neurobiol. Aging 27, 1145-1154. doi: 10.1016/j.neurobiolaging.2005. 06.009

Machann, J., Horstmann, A., Born, M., Hesse, S., and Hirsch, F. W. (2013). Diagnostic imaging in obesity. Best Pract. Res. Clin. Endocrinol. Metab. 27, 261-277. doi: 10.1016/j.beem.2013.02.003

Makrogiannis, S., Boukari, F., and Ferrucci, L. (2018). Automated skeletal tissue quantification in the lower leg using peripheral quantitative computed tomography. Physiol. Meas. 39:035011. doi: 10.1088/1361-6579/aaafb5

McDermott, M. M., Ferrucci, L., Guralnik, J., Tian, L., Liu, K., Hoff, F., et al. (2009). Pathophysiological changes in calf muscle predict mobility loss at 2-year follow-up in men and women with peripheral arterial disease. Circulation 120, 1048-1055. doi: 10.1161/CIRCULATIONAHA.108.842328

Miettinen, T. A. (1971). Cholesterol production in obesity. Circulation 44, 842-850. doi: 10.1161/01.CIR.44.5.842

Miljkovic, I., and Zmuda, J. M. (2010). Epidemiology of myosteatosis. Curr. Opin. Clin. Nutr. Metab. Care 13, 260-264. doi: 10.1097/MCO.0b013e328337d826

Nakagawa, Y., Hattori, M., Harada, K., Shirase, R., Bando, M., and Okano, G. (2007). Age-related changes in intramyocellular lipid in humans by in vivo H-MR spectroscopy. Gerontology 53, 218-223. doi: 10.1159/00010 0869

Newman, A. B., Lee, J. S., Visser, M., Goodpaster, B. H., Kritchevsky, S. B., Tylavsky, F. A., et al. (2005). Weight change and the conservation of lean mass in old age: the Health, Aging and Body Composition study. Am J Clin Nutr 82, 872-8;quiz915-6. doi: 10.1093/ajcn/82.4.872

Noble, J. J., Charles-Edwards, G. D., Keevil, S. F., Lewis, A. P., Gough, M., and Shortland, A. P. (2014). Intramuscular fat in ambulant young adults with bilateral spastic cerebral palsy. BMC Musculoskelet. Disord. 15:236. doi: 10. 1186/1471-2474-15-236

Pahor, M., and Kritchevsky, S. (1998). Research hypotheses on muscle wasting, aging, loss of function and disability. J. Nutr. Health Aging 2, 97-100.

Pan, D. A., Lillioja, S., Kriketos, A. D., Milner, M. R., Baur, L. A., Bogardus, C., et al. (1997). Skeletal muscle triglyceride levels are inversely related to insulin action. Diabetes 46, 983-988. doi: 10.2337/diab.46.6.983

Perseghin, G. (2005). Muscle lipid metabolism in the metabolic syndrome. Curr. Opin. Lipidol. 16, 416-420. doi: 10.1097/01.mol.0000174401.07056.56

Petersen, K. F., Befroy, D., Dufour, S., Dziura, J., Ariyan, C., Rothman, D. L., et al. (2003). Mitochondrial dysfunction in the elderly: possible role in insulin resistance. Science 300, 1140-1142. doi: 10.1126/science.1082889

Prasetyo, M., Nindita, N., Murdana, I. N., Prihartono, J., and Setiawan, S. I. (2020). Computed tomography evaluation of fat infiltration ratio of the multifidus muscle in chronic low back pain patients. Eur. J. Radiol. Open 7:100293. doi: 10.1016/j.ejro.2020.100293
Provencher, S. W. (1993). Estimation of metabolite concentrations from localized in vivo proton NMR spectra. Magn. Reson. Med. 30, 672-679. doi: 10.1002/ mrm.1910300604

Raja, G. K., Sarzynski, M. A., Katzmarzyk, P. T., Johnson, W. D., Tchoukalova, Y., Smith, S. R., et al. (2014). Commonality versus specificity among adiposity traits in normal-weight and moderately overweight adults. Int. J. Obes. (Lond.) 38, 719-723. doi: 10.1038/ijo.2013.153

Schick, F., Eismann, B., Jung, W. I., Bongers, H., Bunse, M., and Lutz, O. (1993). Comparison of localized proton NMR signals of skeletal muscle and fat tissue in vivo: two lipid compartments in muscle tissue. Magn. Reson. Med. 29, 158-167. doi: 10.1002/mrm.1910290203

Schrauwen-Hinderling, V. B., Hesselink, M. K., Schrauwen, P., and Kooi, M. E. (2006). Intramyocellular lipid content in human skeletal muscle. Obesity (Silver Spring) 14, 357-367. doi: 10.1038/oby.2006.47

Schrauwen-Hinderling, V. B., Kooi, M. E., Hesselink, M. K., Jeneson, J. A., Backes, W. H., van Echteld, C. J., et al. (2007). Impaired in vivo mitochondrial function but similar intramyocellular lipid content in patients with type 2 diabetes mellitus and BMI-matched control subjects. Diabetologia 50, 113-120. doi: 10.1007/s00125-006-0475-1

Siparsky, P. N., Kirkendall, D. T., and Garrett, W. E. Jr. (2014). Muscle changes in aging: understanding sarcopenia. Sports Health 6, 36-40. doi: 10.1177/ 1941738113502296

St-Onge, M. P. (2005). Relationship between body composition changes and changes in physical function and metabolic risk factors in aging. Curr. Opin. Clin. Nutr. Metab. Care 8, 523-528. doi: 10.1097/01.mco.0000171150.49 248.14

van der Meer, R. W., Lamb, H. J., Smit, J. W., and de Roos, A. (2012). MR imaging evaluation of cardiovascular risk in metabolic syndrome. Radiology 264, 21-37. doi: 10.1148/radiol.12110772

Visser, M., Goodpaster, B. H., Kritchevsky, S. B., Newman, A. B., Nevitt, M., Rubin, S. M., et al. (2005). Muscle mass, muscle strength, and muscle fat infiltration as predictors of incident mobility limitations in well-functioning older persons. J. Gerontol. A Biol. Sci. Med. Sci. 60, 324-333. doi: 10.1093/gerona/60. 3.324

Visser, M., Kritchevsky, S. B., Goodpaster, B. H., Newman, A. B., Nevitt, M., Stamm, E., et al. (2002). Leg muscle mass and composition in relation to lower extremity performance in men and women aged 70 to 79 : the Health, Aging and Body Composition study. J. Am. Geriatr. Soc. 50, 897-904. doi: 10.1046/j.1532-5415. 2002.50217.x

Weis, J., Johansson, L., Courivaud, F., Karlsson, F. A., and Ahlström, H. (2007). Quantification of intramyocellular lipids in obese subjects using spectroscopic imaging with high spatial resolution. Magn. Reson. Med. 57, 22-28. doi: 10. $1002 / \mathrm{mrm} .21085$

Yokota, T., Kinugawa, S., Yamato, M., Hirabayashi, K., Suga, T., Takada, S., et al. (2013). Systemic oxidative stress is associated with lower aerobic capacity and impaired skeletal muscle energy metabolism in patients with metabolic syndrome. Diabetes Care 36, 1341-1346. doi: 10.2337/dc12-1161

Zane, A. C., Reiter, D. A., Shardell, M., Cameron, D., Simonsick, E. M., Fishbein, K. W., et al. (2017). Muscle strength mediates the relationship between mitochondrial energetics and walking performance. Aging Cell 16, 461-468. doi: 10.1111/acel.12568

Conflict of Interest: The authors declare that the research was conducted in the absence of any commercial or financial relationships that could be construed as a potential conflict of interest.

Copyright (c) 2021 This work is authored by Nicholas A. Brennan, Kenneth W. Fishbein, David A. Reiter, Luigi Ferruci and Richard G. Spencer on behalf of the U.S. Government and, as regards Dr. Brennan, Dr, Fishbein, Dr. Reiter, Dr. Ferruci, Dr. Spencer and the U.S. Government, is not subject to copyright protection in the United States. Foreign and other copyrights may apply. This is an open-access article distributed under the terms of the Creative Commons Attribution License (CC BY). The use, distribution or reproduction in other forums is permitted, provided the original author(s) and the copyright owner(s) are credited and that the original publication in this journal is cited, in accordance with accepted academic practice. No use, distribution or reproduction is permitted which does not comply with these terms. 